\title{
Minimizing biosignal recording sites for hybrid noninvasive brain/neural robot control
}

\author{
Alessia Cavallo, Vincent Roth, David Haslacher, Marius Nann, Surjo R. Soekadar*
}

\begin{abstract}
Noninvasive brain/neural controlled robots are promising tools to improve autonomy and quality of life in severe paralysis, but require biosignal recordings, such as electroencephalography (EEG) and electrooculography (EOG), from various sites distributed over the user's head. This limits the applicability and practicality of noninvasive brain/neural robot control on an everyday basis. It would thus be very desirable to minimize the number of necessary recording sites paving the way for miniaturized, headset-like EEG/EOG systems that users with hemiplegia can mount by themselves. Here, we introduce a novel EEG/EOG brain/neural robot control strategy using only scalp electrodes placed near cortical sensorimotor areas. The strategy was tested across 16 healthy volunteers engaging in an EEG/EOG brain/neural control task. Classification accuracies were compared using scalp electrodes only vs. the conventional electrode placements across the scalp and face. To evaluate whether cranial muscle artifacts impede classification accuracy, participants were asked to chew during the task. We found that brain/neural classification accuracy was comparable and that chewing did not impact classification accuracies when using scalp electrodes only. Our results suggest that the proposed new strategy allows for reliable EEG/EOG-based brain/neural robot control, a critical prerequisite to broaden the use of noninvasive brain/neural assistive and rehabilitative technologies.
\end{abstract}

Index Terms - brain-computer interfaces, electroencephalography, electrooculography, rehabilitation robotics, robot control, wearable sensors

\section{INTRODUCTION}

Introducing brain-controlled robotic devices from the lab into real-world applications is particularly challenging because environmental noise or other signal artifacts can impede classification of brain signals [1][2]. Recently, it was suggested that inclusion of additional physiological signals, e.g. related to eye or muscle movements, can improve safety and reliability of brain-controlled devices, such as active hand exoskeletons, when used outside the lab [3][4].

Besides providing an intuitive control mechanism for assistive applications, it was shown that translation of voluntary modulations of brain activity related to movement attempt or motor imagery into exoskeleton control can trigger neural

Submitted on November $13^{\text {st }} 2019$

This work was supported in part by the European Research Council (ERC) under the project NGBMI (759370), the Baden-Württemberg Stiftung (NEU007/1) and Einstein Stiftung Berlin. SRS received special support by the Brain \& Behavior Research Foundation as 2017 NARSAD Young Investigator Grant recipient and P\&S Fund Investigator.

Alessia Cavallo, Vincent Roth, David Haslacher, Marius Nann and Surjo Soekadar are with the Clinical Neurotechnology Laboratory, Neuroscience Research Center (NWFZ), Department of Psychiatry and Psychotherapy, recovery when used repeatedly [5][6][7]. This rehabilitative approach substantially extends the clinical relevance of braincontrolled devices, because approximately one in three stroke survivors suffers from severe motor impairments for which no other treatment strategy exists [8].

It would thus be very desirable to design brain/neural computer interaction (BNCI) systems that are broadly applicable and can be operated by stroke survivors without any assistance, e.g. in their home environment. This would not only increase the system's impact on the user's quality of life, but also foster generalization of learned skills into the user's reallife environment.

A major obstacle towards this goal, however, relates to the necessary quantity of electrodes for recording biosignals from different recording sites across the user's head. For instance, a well-established noninvasive brain/neural control paradigm uses electroencephalography (EEG) recorded from the scalp region as well as electrooculography (EOG) recorded from the medial or lateral canthus in close proximity to the eyes [9][10]. The lateral canthus is particularly suitable to record horizontal oculoversions (HOV), i.e. maximal horizonal eye movements, because of its proximity to the EOG's electrical source (the corneo-retinal standing potential) and its distance to muscles of mastication (M. masseter, M. temporalis, and M. pterygoideus medialis et lateralis) that are broadly attached to the cranial bones. Electric field modulations related to eye movements recorded at the outer canthus typically range between 50-3500 $\mu \mathrm{V}$ [11] with their amplitudes inversely proportional to the square of the distance from the source [12]. Establishing a strategy that reduces the number and distribution of necessary electrodes, particularly of EOG electrodes placed into the user's face, would pave the way for miniaturized, headset-like EEG/EOG systems that stroke survivors with hemiplegia can mount by themselves. Moreover, eliminating the necessity of electrodes in the facial region may improve user acceptance and therefore adoption and effectiveness. It was unclear, however, whether a reduction in recordings sites, e.g. for detection of $\mathrm{HOV}$, is feasible without compromising brain/neural control performance.

Charité -University Medicine Berlin, Charitéplatz 1, 10117 Berlin, Germany (e-mail: alessia.cavallo@charite.de, roth.vincent.etu@gmail.com, david.haslacher@charite.de, marius.nann@charite.de, surjo.soekadar@charite.de). Marius Nann and Surjo R. Soekadar are additionally with the Applied Neurotechnology Laboratory, Department of Psychiatry and Psychotherapy, University Hospital of Tübingen, Calwerstr. 14, 72076 Tübingen, Germany. *Corresponding author: Surjo R. Soekadar (email: surjo.soekadar@charite.de). 
Here, we investigated whether reliable EEG/EOG brain/neural robot control is feasible and robust using scalp electrodes placed in proximity to the sensorimotor areas only, and whether it provides similar brain/neural control performance as the conventional montage. While we reasoned that with increasing distance from the eyes classification accuracy of HOV would deteriorate and substantially worsen when muscles of mastication are contracted, we hypothesized that combination of several bilateral electrode pairs may compensate for such an effect and result in equivalent control performance.

\section{Materials AND MethodS}

\section{A. Participants}

16 BNCI-naive healthy volunteers ( 7 male, 9 females, mean age: $30.31 \pm 9.24)$ were invited to the Department of Psychiatry and Psychotherapy at the Campus Charite Mitte (CCM) of the Charité - University Medicine Berlin, Germany, to participate in a 2-hour experimental session. All participants were righthanded [13], had no history of neurological or psychiatric disorders and did not take any medication on a regular basis. Before the experiment, all participants provided written informed consent. The study protocol was approved by the Charité's local ethics committee (registration number EA1/077/1).

\section{B. Biosignal recordings}

A custom version of the BCI2000 software platform (www.bci2000.org) was used for signal processing and visual feedback presentation on a computer screen. An active wet electrode system (actiCAP $\AA$ ) coupled with an amplifier unit (LiveAmp ${ }^{\circledR}$, Brain Products GmbH, Gilching, Germany) was used to record and amplify biosignals from the following sites according to the international 10/20 system: F3, F4, T3, T4, C3, $\mathrm{C} 4, \mathrm{P} 3, \mathrm{P} 4$, and $\mathrm{Cz}$ (scalp electrodes). Moreover, in accordance to the standard EOG placements, two electrodes were placed at the left and right outer canthus (facial electrodes). To detect activity of muscles of mastication, two additional electrodes were placed on the right masseter muscle (Fig. 1). A reference electrode was placed at $\mathrm{FCz}$ and a ground electrode was placed at FPz. All biosignals were sampled at $1000 \mathrm{~Hz}$ and high-pass filtered at $0.1 \mathrm{~Hz}$ with a notch-filter at $50 \mathrm{~Hz}$.

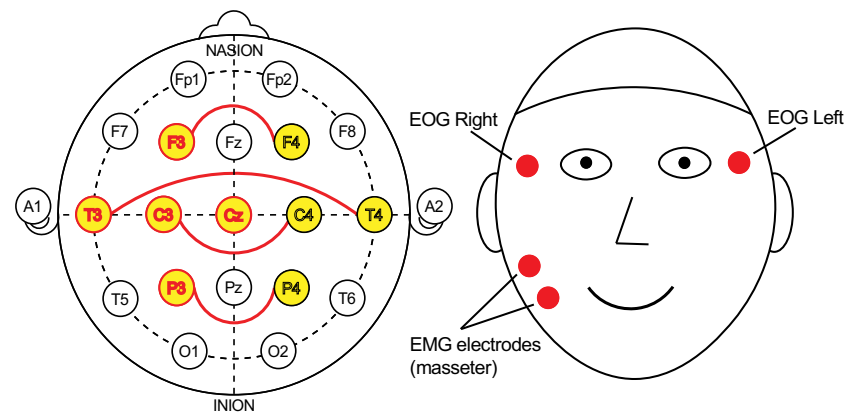

Fig. 1. Distribution of biosignal recording sites across the scalp (left panel) and face (right panel). While signals from electrode sites covering the scalp and face were used in condition1, HOV detection was performed using scalp electrodes only in condition2 (indicated in yellow). To assess activity of muscles of mastication, two additional electrodes were placed over the right M. masseter.

\section{1) Signal processing chain for brain activity ( $\left.S P_{\text {Brain }}\right)$}

For online processing of brain activity, the signals from $\mathrm{F} 3$, T3, C3, P3 and Cz were filtered between 1 and $30 \mathrm{~Hz}$ using a second order Butterworth filter (Fig. 1). A surface Laplacian filter was applied according to [14]. The power spectrum of a $0.4 \mathrm{~s}$ window was computed at $10-12 \mathrm{~Hz}$ using an autoregressive model of order 100 with 15 evaluations per bin. The frequency range was chosen to detect event-related synchronization/ desynchronization (ERS/ERD) of sensorimotor rhythm (SMR) typically occurring during motor imagery or motor planning. The output of this processing chain $\left(\mathrm{SP}_{\mathrm{Brain}}\right)$ was translated into online visual feedback (see section II.C). Before BNCI online control, a calibration run was performed to compute an individualized SMR-ERD detection threshold for each participant.

\section{a) Calibration of the SMR-ERD detection threshold}

Participants were asked to imagine to close their right hand when the word "close" was displayed on a computer screen placed in front of them. When the word "relax" was displayed, participants were instructed to rest. "Close" and "Relax" visual cues were presented 10 times each with a duration of $5 \mathrm{~s}$ followed by an inter-trial interval (ITI) of $4 \mathrm{~s}$. The average output of the SPBrain during all cues was calculated and used as a reference value (RV). To obtain the relative deviation from the RV during each trial, RV was subtracted from the chain output and the result divided by RV. The SMR-ERD detection threshold was then set to the average of the chain output during ten "close" calibration trials and kept unchanged throughout the experiment.

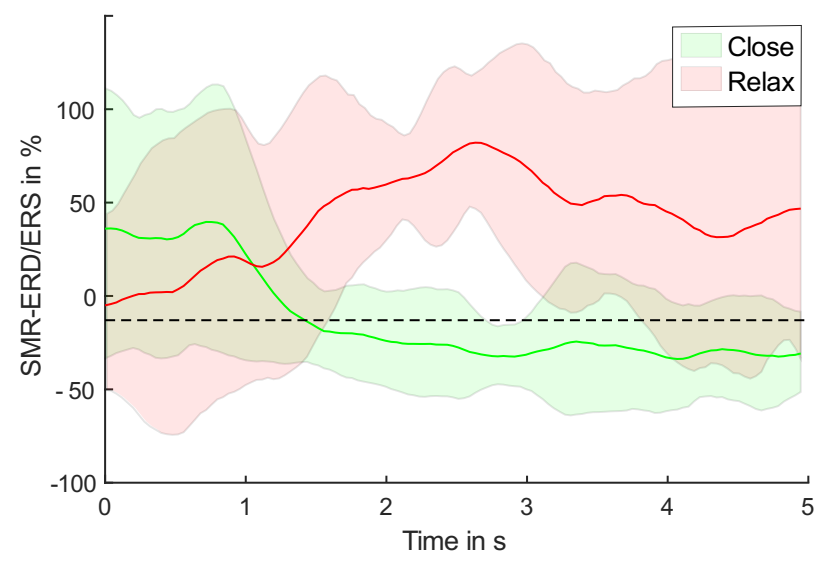

Fig. 2. Illustration of sensorimotor rhythm event-related desynchronization and synchronization (SMR-ERD/ERS) in a representative participant. The red line represents SMR-ERS during the instruction to relax, while the green line represents SMR-ERD during the instruction to imagine hand closing motions. The SMR-ERD detection threshold is indicated as black dotted line.

\section{2) Signal processing chain for $\mathrm{HOV}\left(S P_{\mathrm{HOV}}\right)$}

To detect $\mathrm{HOV}$, four bipolarized electrode pairs were used (F3- F4, T3-T4, C3-C4, P3-P4), subtracting signals from electrodes on the right hemisphere form signal from electrodes on the left hemisphere (Fig. 1). The bipolar signals were then low-pass filtered at $1.5 \mathrm{~Hz}$ using a second order Butterworth 
filter and summed together in order to increase the signal-tonoise ratio. The output of $\mathrm{SP}_{\mathrm{HOV}}$ was translated into visual feedback (see section II.C). Before BNCI online control, a calibration run was performed to calculate the $\mathrm{HOV}$ detection threshold for each participant.

\section{a) Calibration of the HOV detection threshold}

Participants were instructed to perform maximal HOV without turning their head when an arrow to the left or the right was displayed on the computer screen. Each arrow was presented five times for a duration of $2 \mathrm{~s}$ and was followed by an ITI of $4 \mathrm{~s}$. For each arrow to the left (r. right), the maximum (r. minimum) of the $\mathrm{SP}_{\mathrm{Hov}}$ was computed. The detection threshold for left (r. right) HOV was set to two third of the average maximum (r. minimum) chain output and was kept unchanged throughout the experiment.

The calibration runs and subsequent calculation of the SMRERD detection and HOV thresholds were repeated when BNCI control remained below chance level at the end of calibration.

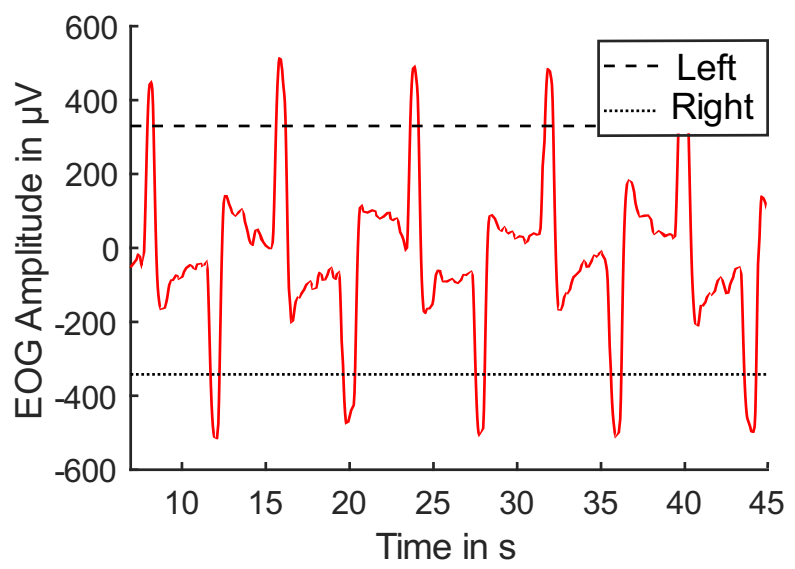

Fig. 3. Illustration of horizontal oculoversions (HOV) to the left (positive deflections) and right (negative deflections) as measured by scalp electrodes only (condition2) in a representative participant. The HOV detection threshold for BNCI control is indicated as a black dashed line for HOV to the left, and a dotted line for HOV to the right.

\section{Testing the BNCI system}

The experimental design included two blocks (block1, block2) with two runs each (run1, run2) comprising a total of 160 trials. While in block1, participants were instructed to relax their cranial muscles, participants were asked to activate muscles of mastication by clenching their teeth in block2. During each run, a sequence of 40 trials was presented. To allow for calculation of sensitivity and specificity of BNCI control, four different visual cues were displayed ten times in a randomized order. Each cue was displayed for $5 \mathrm{~s}$ followed by an ITI of $4 \mathrm{~s}$ (Fig. 4). Before the start of the experiment, participants were familiarized with the experimental design and procedure.

1. During the first cue (cue1, Fig. 4), a yellow half circle was displayed in conjunction with the word "close" to indicate that the participant should imagine right-hand closing motions. If the output of $\mathrm{SP}_{\text {Brain }}$ fell below the SMR-ERD detection threshold, the yellow circle filled by an additional $6 \%$. The yellow circle was fully filled when the SMR-ERD detection threshold was exceeded for more than $3 \mathrm{~s}$.

2. During the second cue (cue2, Fig. 4), a yellow circle accompanied with the text "open" was displayed to indicate that the participant should execute an HOV. If the $\mathrm{SP}_{\mathrm{HOV}}$ exceeded (r. undercut) the HOV detection threshold, the yellow circle changed into a yellow half circle.

3. During the third cue (cue3, Fig. 4), a blue bar was displayed accompanied by the word "relax" to indicate that the participant should rest. If output of the $\mathrm{SP}_{\text {ваn }}$ was below the SMR-ERD detection threshold, the blue bar increased in size, while decreased in size when the output exceeded the threshold.

4. During the fourth cue (cue4, Fig. 4), a yellow circle accompanied by the word "relax" was displayed to indicate that the participant should rest without HOV. If the output of $\mathrm{SP}_{\text {ног }}$ exceeded (r. undercut) the detection threshold for left (r. right) HOV, the yellow circle changed to a yellow half circle.

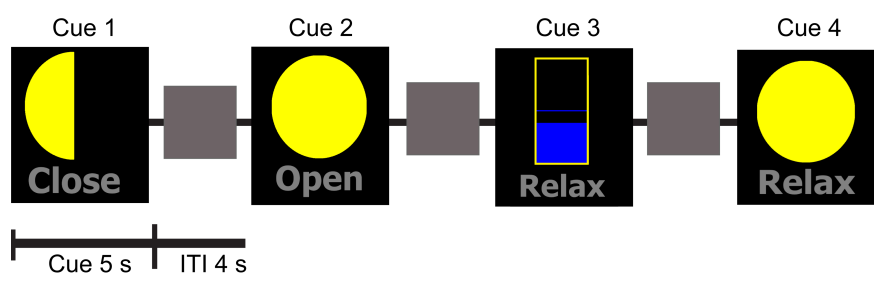

Fig. 4. Task instructions were provided using four different graphical cues. Cuel (left panel) indicated that the participant should engage in motor imagery, cue 2 indicated that the participant should perform a horizontal oculoversions (HOV). Cue 3 and cue4 indicated that participant should rest and relax.

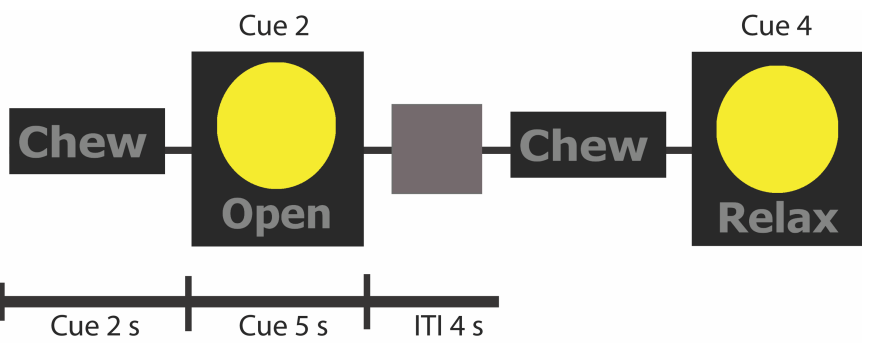

Fig. 5. In block2, an additional visual cue (chew) was used to indicate that the participant should clench the teeth to activate muscles of mastication

\section{Offline data analysis}

The overall BNCI control performance for each participant and block was calculated as the average of the following four performance values:

1. The BNCI system's sensitivity (true positive rate) for SMR-ERD detection was computed as the mean percentage of yellow circle fillings achieved during 20 
presentations of cue1 ("close" instruction accompanied by showing a yellow half circle).

2. The BNCI system's sensitivity for HOV detection was computed as the percentage of circles that changed from a full to a half yellow circle during 20 presentations of cue 2 ("open" instruction accompanied by showing a yellow full circle).

3. The BNCI system's specificity (true negative rate) for SMR-ERD detection was computed as the mean percentage of time the blue bar decreased in size during 20 presentations of cue 3 ("relax" instruction accompanied by showing a blue bar).

4. The BNCI system's specificity for HOV detection was computed as the percentage of trials in which yellow full circles that did not switch to yellow half circles during 20 presentations of cue4 ("relax" instruction accompanied by showing a yellow full circle).

To ensure that during block 2 only trials were included in which participants activated their muscles of mastication, only trials were included in which EMG signals $(10-100 \mathrm{~Hz})$ that were recorded over the right masseter exceeded two standard deviations of EMG signals recorded during block1. To calculate the overall control performance when using the conventional electrode montage, i.e. scalp and face electrodes vs. scalp electrodes alone, BNCI control performance was calculated offline for both conditions (condition1: scalp \& face, condition2: scalp only). In analogy to SPноv described in II.C.2, corresponding electrodes were bipolarized and signals filtered from 0.1 to $1.5 \mathrm{~Hz}$ using a second order Butterworth filter. The threshold for left (r. right) HOV detection was then calculated using the calibration run. The BNCI system's sensitivity and specificity for $\mathrm{HOV}$ detection in condition 1 and condition2 as well as the overall BNCI system's control performance was then computed offline.

To test whether the overall control performance in condition1 was inferior to condition2, a one sample-paired t-test was performed for block 1 and block2. Non-inferiority was assumed at a difference smaller than $2 \%$ with $p<0.05$.

\section{RESULTS}

After familiarization with the task, all participants were able to voluntarily generate SMR-ERD and HOV for brain/neural control. In block 2 , in average $2.01 \pm 1.7 \%$ of cue 2 and cue 4 were discarded due to insufficient masseter activity during the trials.

In block 1, there was no difference in overall BNCI control performance between condition 1 and condition $2(\mathrm{p}<0.001)$ (Fig. 6). Overall BNCI control performance in condition1 was $87.68 \pm 31.48 \%$ and $87.91 \pm 31.88 \%$ in condition 2 indicating that EEG/EOG detection was comparable when using scalp electrodes only as compared to the conventional electrode montage including scalp and face areas.

There was no difference in BNCI control performance in block2 in which participants engaged in mastication $(\mathrm{p}<0.001)$ (Fig.7). Overall BNCI control performance reached $87.17 \pm$ $32.52 \%$ with scalp \& facial electrodes (condition1) and $86.74 \pm$ $39.12 \%$ with scalp electrodes only (condition2) (Fig. 7).
Under condition1, sensitivity and specificity of $\mathrm{HOV}$ detection across all participants and blocks reached $100 \%$. Under condition2, 100\% sensitivity and specificity of HOV detection was reached by 15 participants in block1 and 14

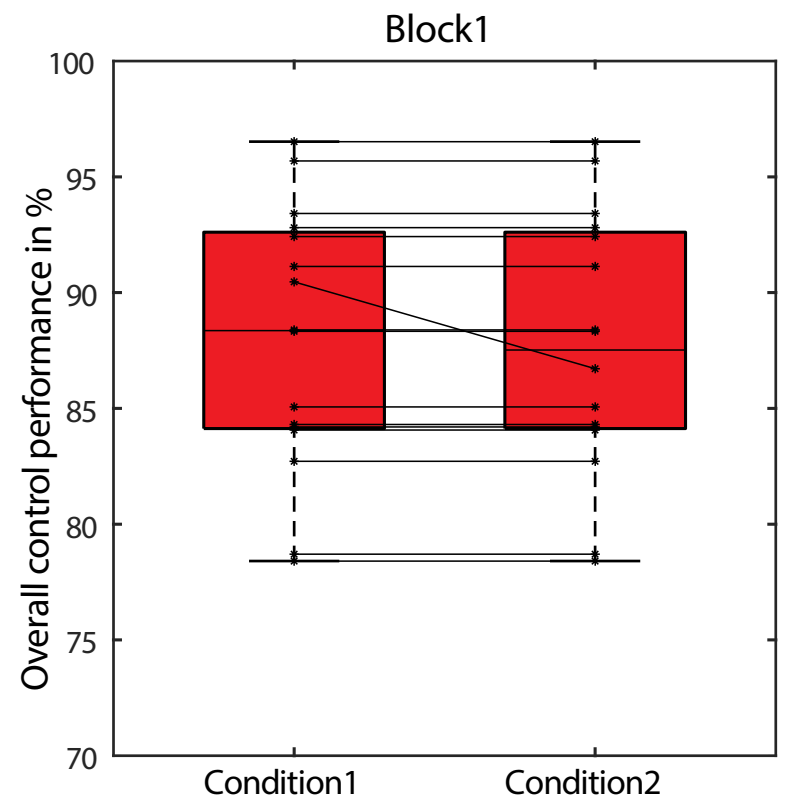

Fig. 6. Overall BNCI control performance across all study participants in block1 under condition1 (using biosignals from electrode sites distributed over the scalp and face) (left) and condition2 (using biosignals from the scalp region only) (right). Changes in BNCI performance between conditions is shown as black line for each participant. The median is shown as block line in each box graph, the 25 th and 75 th percentiles are indicated by the error bars.

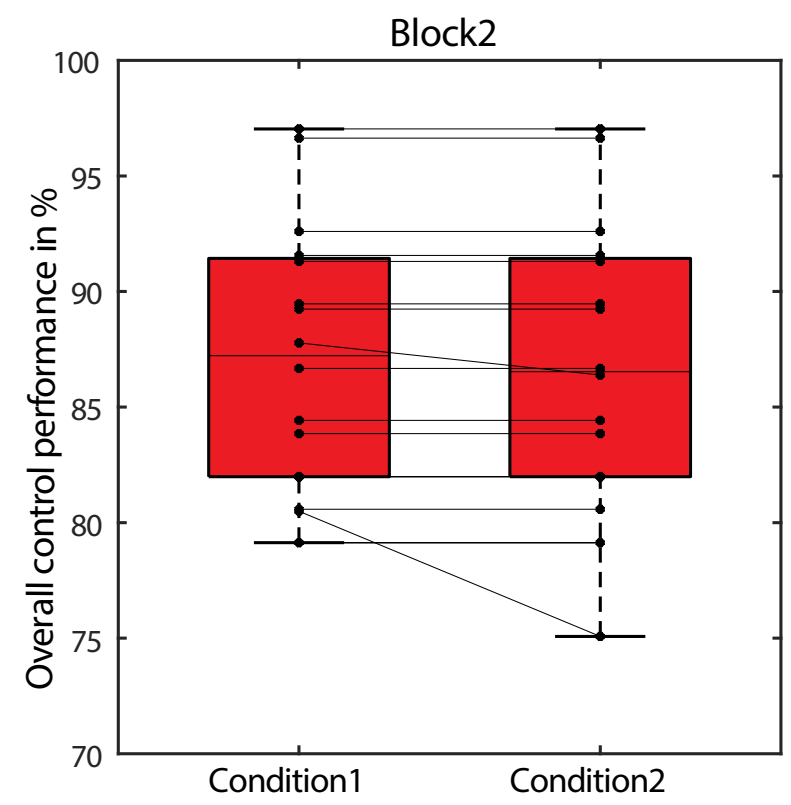

Fig. 7. Overall BNCI control performance across all study participants in block2 under condition1 (using biosignals from electrode sites distributed over the scalp and face) (left) and condition2 (using biosignals from the scalp region only) (right). Changes in BNCI performance between conditions is shown as black line for each participant. The median is shown as block line in each box graph, the 25 th and 75 th percentiles are indicated by the error bars. 
participants in block2. While specificity of HOV detection was $100 \%$ across all participants, sensitivity was $85 \%$ in block 1 of one participant, and $95 \%$ and $78 \%$ respectively in block 2 (see Fig. 7).

\section{DISCUSSION}

Reducing the number of biosignal recording sites for noninvasive hybrid brain/neural robot control is important to broaden applicability of assistive and rehabilitative BNCI systems. Here, we tested a strategy for reliable EEG/EOG brain/neural control that exploits the fact that the eye's electric fields spread over the scalp and can be detected over the brain's sensorimotor areas [12]. It was unclear, however, whether online HOV detection can be achieved at similar accuracies using this strategy compared to the established approach recording HOV from the outer canthus [4][9][10][15]. We found that $\mathrm{EEG} / \mathrm{EOG}$ classification accuracies were comparable when using scalp electrodes placed in proximity to the sensorimotor areas only (condition2) as compared to the conventional approach with additional electrodes placed in the facial area at the outer canthus (condition1). Robustness of BNCI classification in each condition was evaluated by comparing classification accuracies with study participants relaxing their jaw (block1) vs. activating their muscles of mastication by clenching their teeth (block2). Independent of masseter contractions, classification accuracies were comparable across blocks indicating that the new strategy is robust even when engaging in mastication involved in various activities of daily living, such as eating, drinking or speaking. While specificity of HOV detection was $100 \%$ across all participants, blocks and conditions, sensitivity was $100 \%$ for 15 participants under condition 2 in block1 and 14 participants in block2. The reason for this reduction in sensitivity in block2 found in two participants was most likely due to a rather conservative adjustment of the HOV detection threshold. Given that specificity was $100 \%$ across all participants, it is conceivable that setting the HOV detection threshold not at two third of the average maximum or minimum of SPноv, but slightly below that (e.g. at 60\%), would result in $100 \%$ sensitivity across all participants without affecting specificity of HOV detection. This issue should be further investigated in follow-up studies.

While this study focused on reducing the number of head areas required for reliable BNCI control (scalp \& face vs. scalp only), it is conceivable that further reduction of the number and distribution of electrode recording sites is possible. Here, use of tri-polar concentric ring electrodes could be a viable solution [16][17]. It was suggested that individualization of EEG electrode sites may improve classification accuracies [9], particularly in patients who suffered a stroke [18] or other conditions associated with cortical reorganization [19]. In this context, it will be important to assess the impact of such individualization on EEG/EOG classification accuracies when using the proposed strategy. Also, it is unclear how different disease conditions that are associated with eye movement disorders, e.g. Parkinson's or multiple sclerosis [20], affect reliability of the proposed approach.

Besides minimizing the number of recording sites, also other factors will influence applicability and user acceptance of
BNCI systems. Here, wearing comfort and a non-stigmatizing overall design are key elements to increase adoption of BNCI technology into everyday life environments. Future assistive and rehabilitative BNCI systems should be as intelligent, but also as intuitive and simple as possible [21]. Moreover, various neuroethical dimensions have to be considered when bringing BNCI technology out of the lab into real world applications [22].

Besides improving neuroliteracy, ensuring accountability, responsibility, privacy and security are important dimensions on this path. The proposed BNCI control strategy specifically contributes to the dimension of accountability, because it provides a reliable option to, e.g., stop (veto) an unwanted action of a brain-controlled actuator. Such veto function is particularly essential for shared control paradigms, in which a human interacts with an autonomous robotic system [23]. The proposed veto function could be easily implemented in existing EEG-based BCI paradigms without the necessity of adding EOG electrodes in the facial region and thus increase their applicability in real-world scenarios. Further validation of this approach in clinical studies involving patient populations will be necessary, however.

\section{REFERENCES}

[1] J. A. Uriguen and B. Garcia-Zapirain, "EEG artifact removal-state-ofthe-art and guidelines," J Neural Eng, vol. 12, no. 3, p. 031001, Jun 2015.

[2] M. K. Islam, A. Rastegarnia, and Z. Yang, "Methods for artifact detection and removal from scalp EEG: A review," Neurophysiol Clin, vol. 46, no. 4-5, pp. 287-305, Nov 2016.

[3] M. Witkowski, M. Cortese, M. Cempini, J. Mellinger, N. Vitiello, and S. R. Soekadar, "Enhancing brain-machine interface (BMI) control of a hand exoskeleton using electrooculography (EOG)," J Neuroeng Rehabil, vol. 11, p. 165, Dec 162014.

[4] S. R. Soekadar, M. Witkowski, N. Vitiello, and N. Birbaumer, "An EEG/EOG-based hybrid brain-neural computer interaction (BNCI) system to control an exoskeleton for the paralyzed hand," Biomed Tech (Berl), vol. 60, no. 3, pp. 199-205, Jun 2015.

[5] A. Ramos-Murguialday et al., "Brain-machine interface in chronic stroke rehabilitation: a controlled study," Ann Neurol, vol. 74, no. 1, pp. 100-8, Jul 2013.

[6] A. R. Donati et al., "Long-Term Training with a Brain-Machine Interface-Based Gait Protocol Induces Partial Neurological Recovery in Paraplegic Patients," Sci Rep, vol. 6, p. 30383, Aug 112016.

[7] M. A. Cervera et al., "Brain-computer interfaces for post-stroke motor rehabilitation: a meta-analysis," Ann Clin Transl Neurol, vol. 5, no. 5, pp. 651-663, May 2018.

[8] V. Feigin et al., "Global Burden of Diseases, Injuries and Risk Factors Study 2013 and Stroke Experts Writing Group. Global burden of stroke and risk factors in 188 countries, during 1990-2013: a systematic analysis for the Global Burden of Disease Study 2013," Lancet Neurol, vol. 15, no. 9, pp. 913-924, 2016.

[9] S. R. Soekadar et al., "Hybrid EEG/EOG-based brain/neural hand exoskeleton restores fully independent daily living activities after quadriplegia," Science Robotics, 10.1126/scirobotics.aag3296 vol. 1, no. $1,2016$.

[10] M. Witkowski, M. Cortese, M. Cempini, J. Mellinger, N. Vitiello, and S. R. Soekadar, "Enhancing brain-machine interface (BMI) control of a hand exoskeleton using electrooculography (EOG)," J Neuroeng Rehabil, journal article vol. 11, no. 1, p. 165, Dec 162014.

[11] M. Akay, Wiley encyclopedia of biomedical engineering. WileyInterscience, 2006

[12] R. J. Croft and R. J. Barry, "Removal of ocular artifact from the EEG: a review," Neurophysiol Clin, vol. 30, no. 1, pp. 5-19, Feb 2000.

[13] R. C. Oldfield, "The assessment and analysis of handedness: the Edinburgh inventory," Neuropsychologia, vol. 9, no. 1, pp. 97-113, Mar 1971. 
[14] C. Carvalhaes and J. A. de Barros, "The surface Laplacian technique in EEG: Theory and methods", Int. J. Psychophysiol., vol. 97, no. 3, pp. 174188, May 2015.

[15] S. Crea, Nann, M., Trigili, E., Cordella, J.N., J., Baldoni, A., Badesa, F., Catalan, F., Zollo, L., Vitiello, N., Aracil, N.G., Soekadar, S.R., "Feasibility and safety of shared EEG/EOG and vision-guided autonomous whole-arm exoskeleton control to perform activities of daily living," Sci Rep, vol. (in press), 2018.

[16] K. Koka and W. G. Besio, "Improvement of spatial selectivity and decrease of mutual information of tri-polar concentric ring electrodes," J Neurosci Methods, vol. 165, no. 2, pp. 216-22, Sep 302007.

[17] W. G. Besio, K. Koka, R. Aakula, and W. Dai, "Tri-polar concentric ring electrode development for laplacian electroencephalography," IEEE Trans Biomed Eng, vol. 53, no. 5, pp. 926-33, May 2006.

[18] E. Buch et al., "Think to move: a neuromagnetic brain-computer interface (BCI) system for chronic stroke," Stroke, vol. 39, no. 3, pp. 910-7, Mar 2008.

[19] D. T. Pruitt et al., "Traumatic Brain Injury Occludes TrainingDependent Cortical Reorganization in the Contralesional Hemisphere," J Neurotrauma, vol. 34, no. 17, pp. 2495-2503, Sep 2017.

[20] V. T. Lal, Daniel, "Eye movement abnormalities in movement disorders," Clinical Parkinsonism \& Related Disorders, vol. 1, pp. 54 63, 2019.

[21] S. Hazubski, S. R. Soekadar, H. Hoppe, and A. Otte, "Neuroprosthetics 2.0," EBioMedicine, Oct 52019.

[22] J. Clausen et al., "Help, hope, and hype: Ethical dimensions of neuroprosthetics," Science, vol. 356, no. 6345, pp. 1338-1339, Jun 30 2017.

[23] S. Crea et al., "Feasibility and safety of shared EEG/EOG and visionguided autonomous whole-arm exoskeleton control to perform activities of daily living," Sci Rep, vol. 8, no. 1, p. 10823, Jul 172018. 